\title{
Influence of Left Atrial Geometry on Rotor Core Trajectories in a Model of Atrial Fibrillation
}

\author{
Konstantinos N Tzortzis, Caroline H Roney, Norman A Qureshi, Fu Siong Ng, Phang Boon Lim, \\ Spencer J Sherwin, Nicholas S Peters, Chris D Cantwell \\ Imperial College London, London, United Kingdom
}

\begin{abstract}
Left atrial anatomy and myocardial architecture are known to influence rotor initiation and maintenance. However, identifying their relative contribution clinically is challenging. The present study aims to investigate in silico the effect of left atrial geometry in isolation on rotor generation and evolution through the spatiotemporal tracking of phase singularities. After meandering for a short period of time, rotors are attracted to specific areas of the chamber where there is high curvature, primarily near the base of the left atrial appendage and the junctions of the pulmonary veins. This suggests that the left atrial anatomy could play a key role in the perpetuation of fibrillatory activity.
\end{abstract}

\section{Introduction}

Atrial fibrillation (AF) is the most common type of cardiac arrhythmia. One theory about AF maintenance is the presence of localized sources termed rotors [1]. A rotor is defined as a high frequency curved wavefront, which meets the wavetail at a single point and is characterised by the absence of refractory tissue at the centre [2]. Until recently, the presence of rotors was only observed in animal experimental studies [3], but localized and stable rotors in human AF have now been identified $[4,5]$. This progress may increase the efficacy of targeted left atrial ablation therapy.

Clinical studies [6] and human myocardium-based computational studies [7] suggest that rotors may be constrained temporarily or persistently in limited areas of the LA, because of discontinuities in the fibre architecture. In addition, human atria exhibit fibrosis and scar outside the pulmonary veins (PV) to a considerable degree, which may contribute to the initiation mechanisms for AF in many patients [8]. A variety of computational models propose that fibre orientation, fibrosis and the geometry of LA together can play an important role for the generation of anisotropic conductivity $[9,10]$.
Clinical studies show that LA size and diameter and the PV structural characteristics in isolation [11-12] are related with the outcome of catheter ablation and are useful as independent predictors of AF recurrence. In this paper, we investigate, using a computational model, and anatomically accurate LA geometries derived from persistent AF patients, the effect of geometry in isolation on the maintenance of localised fibrillatory propagation, the spatiotemporal evolution of rotors over time and their association with anatomical characteristics.

\section{Methods}

\subsection{Mesh generation}

The closed three-dimensional LA endocardial surface geometry was segmented from late-gadolinium enhanced magnetic resonance imaging (LG-MRI) data, using ITKSNAP [13]. The mitral valve and the PV sleeves were opened in order to produce a topologically accurate finite element mesh of the atrial chamber and PV sleeves. Gmsh [14], a high-order three-dimensional finite element mesh generator, was used to remesh the surface using a coarser uniform triangulation, which consisted of elements of approximately the same characteristic length of a size appropriate for use with a high-order spectral element discretisation [10]. A second mesh, which was used for generating a two-dimensional unwrapping of the surface, was created by adding cuts on either side of the atrium from the mitral valve up to the ends of the veins.

\subsection{Simulation Protocol}

Simulations, using the uncut finite element mesh were performed using the Nektar++ high-order spectral $/ h p$ element framework [16], in which the solution on each mesh element is approximated using a basis of high-order polynomials. Spectral $/ h p$ element methods support convergence of the solution through both mesh refinement and enrichment of the polynomial space, permitting accurate representation of propagating action 

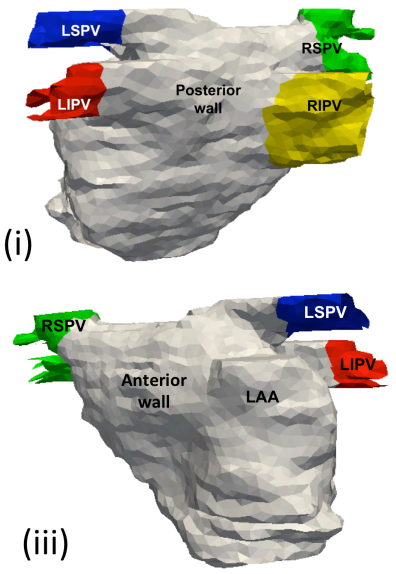

(ii)
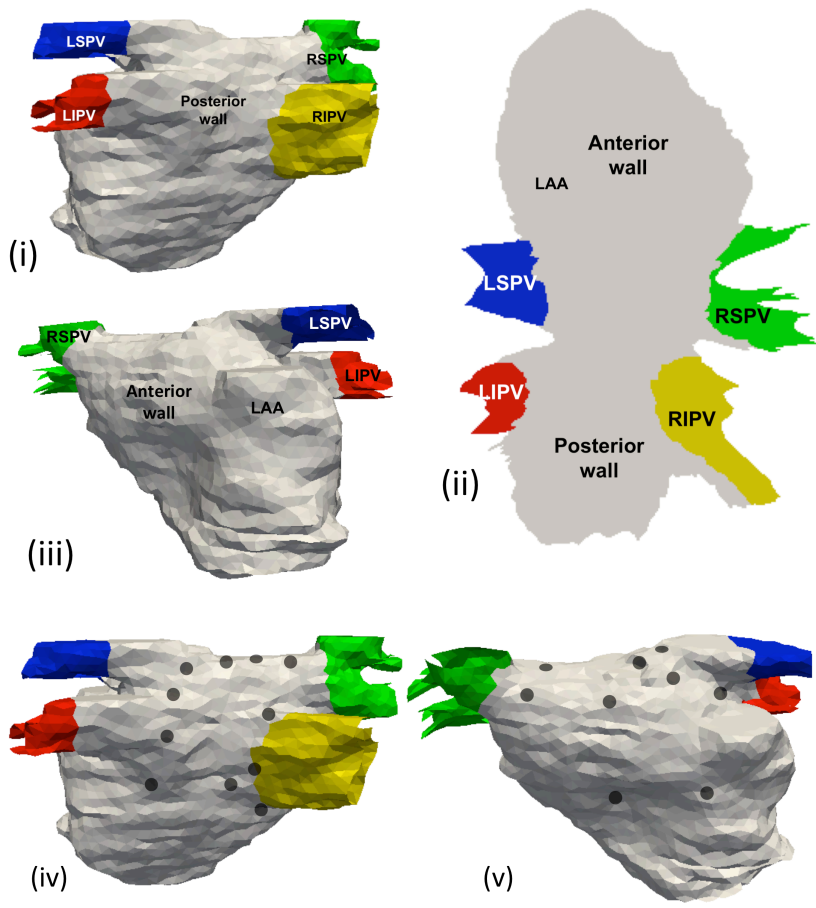

Figure 1. (i-iii) Representative mapping of a left atrium derived from $\mathrm{AF}$ patient to a two-dimensional surface representation. The left superior (blue), left inferior (red), right superior (green) and right inferior (yellow) pulmonary veins and the left atrial appendage are indicated on the MRI-derived geometries. (iv-v) Schematic representation of S2 stimuli on the 3D LA surface, as they were distributed on the posterior (iv) and anterior (v) walls for the performance of a set of twenty simulations.

potential wavefronts with fewer degrees of freedom than conventional linear finite element methods. The monodomain model for describing electrical propagation in cardiac tissue and the Courtemanche-Ramirez-Nattel (CRN) human atrial cell model [17] were used. The CRN model includes modifications representative of the effects of $\mathrm{AF}$ on human action potential properties, for biophysically accurate cellular dynamics.

For the purpose of this study, fibre architecture was ignored. The tissue architecture was prescribed as isotropic with a fixed conductivity of $0.13342 \mathrm{~S} / \mathrm{m}$. Evolution of spiral waves was investigated through an ensemble of twenty simulations, paced at uniformly distributed locations throughout each model. These initial stimulus locations were placed both around the PVs and throughout the anteroposterior area. For each pacing location, an S1-S2 extra stimulus protocol was applied. A wide-area S1 stimulus of $50 \mu \mathrm{A} / \mathrm{mm}^{3}$ was followed 315 ms later by a second smaller S2 stimulus of strength $100 \mu \mathrm{A} / \mathrm{mm}^{3}$ on the boundary of the $\mathrm{S} 1$ stimulus to initiate two counter-rotating spiral waves. The area of the S1

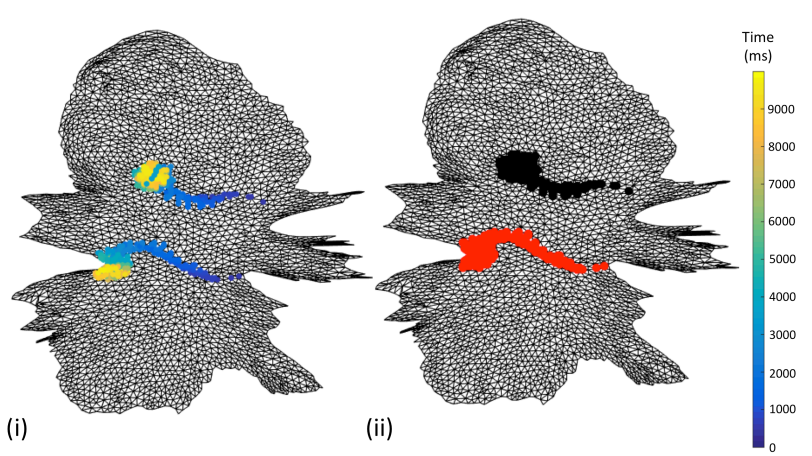

Figure 2. Illustration of a representative pair of phase singularity trajectories (i) and its analogue according to the chirality of phase singularities (ii) for two rotors generated from the same pair of S1-S2 stimuli. The colour code reflects the time point when a phase singularity was detected.

stimulus was chosen sufficiently large to ensure the rotors were self-sustaining.

\subsection{Phase mapping}

The three-dimensional LA surface was unwrapped into a two-dimensional representation [18] as illustrated in Figure 1. This transformation aims to preserve local distances and therefore minimize distance distortions allowing rotor trajectories to be more readily analysed. Voltage data generated through simulation were mapped to the planar surface and interpolated to a regular grid. The phase of the voltage data for each point of the regular grid was calculated and phase singularities were subsequently identified using the topological charge technique [19]. The movement of phase singularities was tracked over time to identify rotor core trajectories.

\section{Results}

The stimulus protocol in each simulation resulted in the formation of a pair of phase singularities with opposite chirality (Figure 2ii). The corresponding rotors meandered on the 3D surface (Figure 2i) and were primarily attracted by the left atrial appendage (LAA) and the PVs (Figures 2-3), where they became spatially stable and meandered only within a small region. The mean distance travelled by rotors from the S2 stimulus point to the centroid point of the area of stability differed between meshes $(\mathrm{P}=0.0465)$ (Table 1). Furthermore, there was no correlation between distance travelled by rotors and surface size $(r=-0.06557,95 \% \mathrm{CI}=-0.255$ to 0.1287 , $\mathrm{P}=0.4961)$.

Rotor trajectories were observed to be sensitive to the initial stimulus location. Figure 3 shows three simulations 


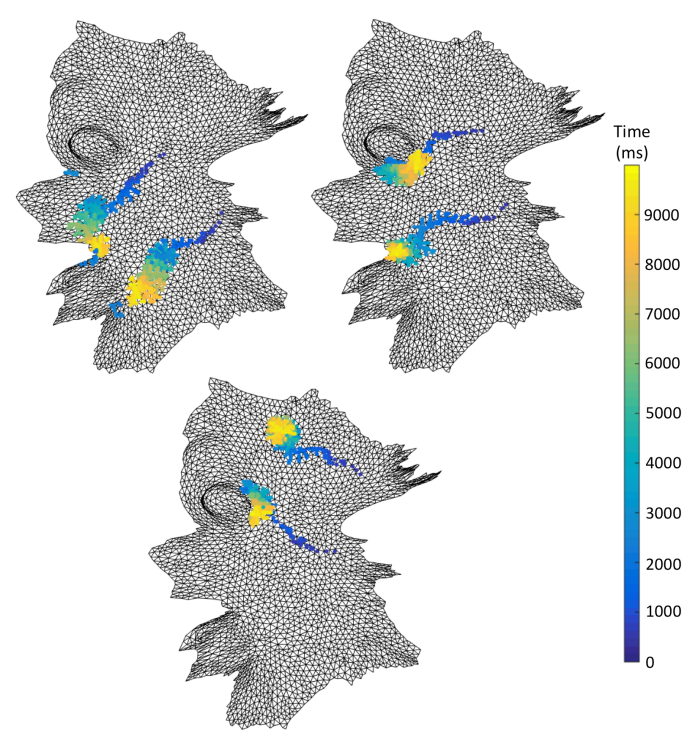

Figure 3. Time-space rotor trajectories on the same 2D mesh surface where is presented that stimulating adjacent locations can lead to alteration of the direction of rotor meandering.

Table 1. One-way ANOVA analysis for comparison of the average distance travelled by rotors in each $3 \mathrm{D}$ mesh surface until they end up in a particular area. Surface size calculated using MeshLab. $\mathrm{N}=$ number of rotor trajectories

\begin{tabular}{cccc}
\hline $\begin{array}{c}\text { \# mesh } \\
(\text { surface size) }\end{array}$ & $\begin{array}{c}\text { Mean distance } \pm \text { S.D. } \\
(\mathrm{mm})\end{array}$ & $\mathrm{N}$ & $P$ \\
\hline $1\left(81.6 \mathrm{~cm}^{2}\right)$ & $51.63 \pm 21.76$ & 36 & \\
$2\left(62.6 \mathrm{~cm}^{2}\right)$ & $57.1 \pm 24.78$ & 38 & 0.0465 \\
$3\left(66.2 \mathrm{~cm}^{2}\right)$ & $44.71 \pm 16.72$ & 37 & \\
\hline
\end{tabular}

of the spatiotemporal evolution of rotors initiated near the RSPV. These examples show significant variation in the trajectories of the rotors and consequently the location within the LA in which they stabilize.

To identify regions of rotor attraction and relate these to anatomical properties of the chamber geometry, a phase singularity distribution map was constructed for each patient and shown in Figure 4. These show the number of phase singularities detected per second for each mesh node of the 2D representation. Rotors are attracted by and typically remain stable in areas of higher geometrical curvature. These are often locations between the LAA and the left superior PV, such as shown in Figures $4 \mathrm{i}$ and iv, and between two adjacent PVs (Figure $4 \mathrm{i}$, iv) or around the LAA (Figure 4). Rotors were occasionally attracted by the PV sleeve, in particular the right superior and inferior PVs.

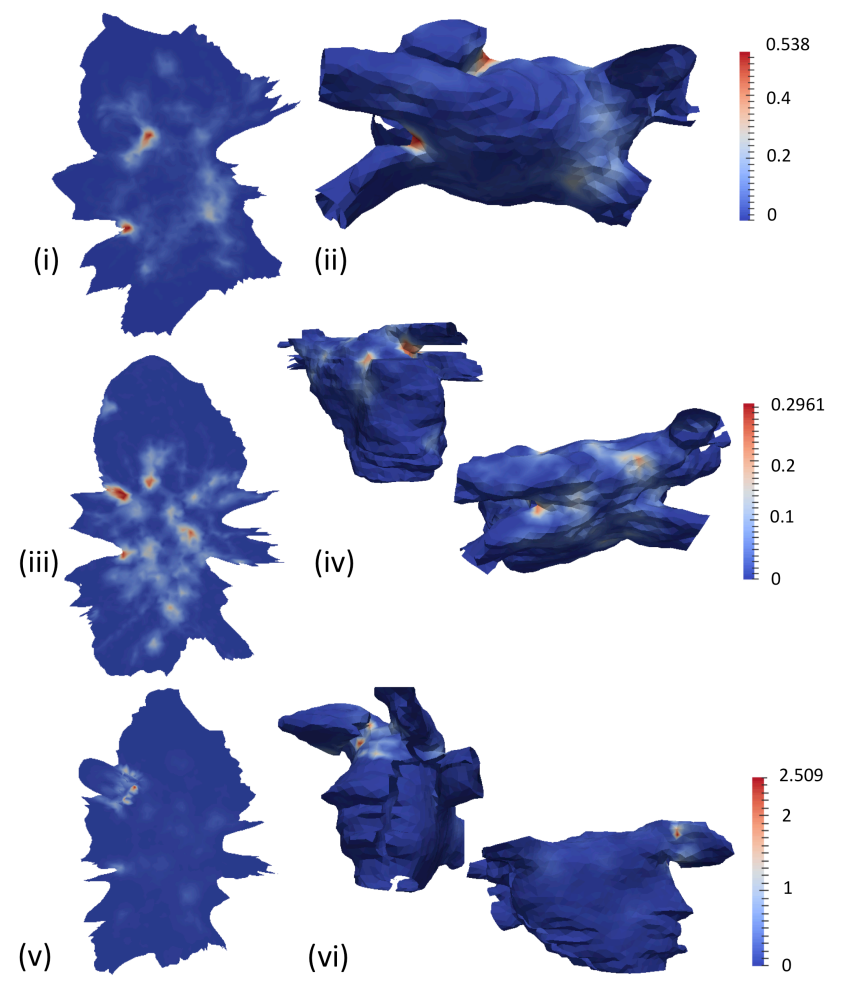

Figure 4. 2D heat maps (i, iii, v) of the investigated meshes for quantification of the LA areas which are more prone to rotor attraction. Figures 4ii, iv, vi correspond to the $3 \mathrm{D}$ representation of $2 \mathrm{D}$ meshes presenting different view angles of the posterior and anterior walls where rotor attraction areas are visible. The colour code reflects the number of phase singularities per second measured at the grid point closest to a vertex.

\section{Discussion}

This study provides insight into the influence of LA structure on rotor evolution. Rotors were detected and their trajectories tracked through the identification of phase singularities in the voltage. Stimulation locations were observed to affect the trajectories of rotors and the location where they ultimately became spatially stable. These areas are predominantly identified around the LAA, between the left superior PV and the LAA or between two adjacent PVs. These are consistently areas with high geometric curvature.

The stimulation protocol used in this study consistently produced a pair of rotors, which will necessarily interact with each other, potentially influencing their trajectories. However, we were unable to reliably generate a single rotor under isotropic conditions to assess this. Another limitation in the present study is that the LAA of one patient is particularly large (Figure $4 \mathrm{v}$ ) and cannot be flattened completely. This may have distorted the phase singularity analysis, although analysis of the voltage data 
suggests the heat map is qualitatively accurate.

The conclusions drawn in this study are predominantly observational and based on a small cohort of three atrial geometries. In future work we will increase the number of patients in the cohort, as well as perform a qualitative comparison with the curvature of the surface. Subsequently, we will apply the same experimental conditions and analysis to a model augmented with fibrosis and/or fibre architecture, in order to investigate the relative contributions of LA geometry and tissue micro-architecture on the maintenance of fibrillatory conduction.

\section{Acknowledgements}

The British Heart Foundation (BHF), grants
RE/13/4/30184 and RG/10/11/28457, the
ElectroCardioMaths Programme of the Imperial BHF
Centre of Research Excellence and the NIHR Imperial
Biomedical Research Centre, supported this work. CDC
acknowledges support from EPSRC, grant EP/L000407/1.

\section{References}

[1] Vaquero M, Calvo D, Jalife J. Cardiac fibrillation: from ion channels to rotors in the human heart. Heart Rhythm 2008;5(6):872-879.

[2] Pandit SV, Jalife J. Rotors and the dynamics of cardiac fibrillation. Circulation research 2013;112(5):849-862.

[3] Cherry EM, Hastings HM, Evans SJ. Dynamics of human atrial cell models: Restitution, memory, and intracellular calcium dynamics in single cells. Progress in biophysics and molecular biology 2008;98(1):24-37.

[4] Narayan SM, Krummen DE, Rappel WJ. Clinical mapping approach to diagnose electrical rotors and focal impulse sources for human atrial fibrillation. Journal of cardiovascular electrophysiology 2012;23(5):447-454.

[5] Haissaguerre M, Hocini M, Shah AJ, Derval N, Sacher F, Jais P, Dubois R. Noninvasive panoramic mapping of human atrial fibrillation mechanisms: a feasibility report. Journal of cardiovascular electrophysiology 2013;24(6):711-7.

[6] Sánchez-Quintana D, Anderson RH, Carbera JA, Climent V, Martin R, Farré J, Ho SY. The terminal crest: morphological features relevant to electrophysiology. Heart 2002;88(4):406-11.

[7] Gonzalez MJ, Vincent KP, Rappel WJ, Narayan SM, McCulloch AD. Structural contributions to fibrillatory rotors in a patient-derived computational model of the atria. Europace 2014;16 Suppl 4 iv3-iv10.

[8] Marrouche NF, Wilber D, Hindricks G, Jais P, Akoum N, Marchlinski F, Kholmovski E, Burgon N, Hu N, Mont L, Deneke T, Duytchaever M, Neumann T, Mansour M, Mahnkopf C, Herweg C, Daoud E, Wissner E, Bansmann $\mathrm{P}$, Brachmann J. Association of atrial tissue fibrosis identified by delayed enhancement MRI and atrial fibrillation catheter ablation: the DECAAF study. JAMA 2014;311(5):498-506.

[9] Aslanidi OV, Colman MA, Varela M, Zhao J, Smaill BH,
Hancox JC, Boyett MR, Zhang H. Heterogeneous and anisotropic integrative model of pulmonary veins: computational study of arrhythmogenic substrate for atrial fibrillation. Interface focus 2013;3(2) 20120069.

[10] Cantwell CD, Yakovlev S, Kirby RM, Peters NS, Sherwin SJ. High-order spectral/element discretisation for reactiondiffusion problems on surfaces: Application to cardiac electrophysiology. Journal of computational physics 2014;257(PA):813-829.

[11] Mansour M, Refaat M, Heist EK, Mela T, Cury R, Holmvang G, Ruskin JN. Three-dimensional anatomy of the left atrium by Magnetic Resonance Angiography: Implications for catheter ablation for atrial fibrillation. Journal of cardiovascular electrophysiology 2006;17(7):719-23.

[12] den Uijl DW, Tops LF, Delgado V, Schuijf JD, Kroft LJM, de Roos A, Boersma E, Trines SA, Zeppenfeld K, Schalij MJ, Bax JJ. Effect of pulmonary vein anatomy and left atrial dimensions on outcome of circumferential radiofrequency catheter ablation for atrial fibrillation. The Americal Journal of Cardiology 2011;107:243-249.

[14] Geuzaine C, Remacle J. Gmsh: A 3-D finite element mesh generator with built-in pre- and post-processing facilities. International Journal for Numerical Methods in Engineering 2009;79(11):1309-1331.

[15] Yushkevich PA, Piven J, Hazlett HC, Smith RG, Ho S, Gee JC, Gerig G. User-guided 3D active contour segmentation of anatomical structures: Significantly improved efficientcy and reliability. NeuroImage 2006;31:1116-28.

[16] Cantwell CD, Moxey D, Comerford A, Bolis A, Rocco G, Mengaldo G, De Grazia D, Yakovlev S, Lombard J-E, Ekelschot D, Jordi B, Xu H, Mohamied Y, Eskilsson C, Nelson B, Vos P, Biotto C, Kirby RM, Sherwin SJ. Nektar++: An open-source spectral $/ h p$ element framework. Computer Physics Communications 2015;192:205-219.

[17] Courtemanche M, Ramirez RJ, Nattel S. Ionic targets for drug therapy and atrial fibrillation-induced electrical remodeling: insights from a mathematical model. Cardiovascular research 1999;42(2):477-489.

[18] Roney CH, Tzortzis KN, Cantwell CD, Qureshi NA, Ali RL, Lim PB, Siggers JH, Ng FS, Peters NS. A technique for visualising three-dimensional left atrial cardiac activation data in two dimensions with minimal distance distortion. $37^{\text {th }}$ Annual International Conference of the IEEE Engineering in Medicine and Biology Sciences 2015.

[19] Bray M-A, Wikswo JP. Use of topological charge to determine filament location and dynamics in a numerical model of scroll wave activity. IEEE transactions on biomedical engineering 2002;49(10):1086-93.

Address for correspondence:

Konstantinos N Tzortzis

National Heart and Lung Institute, Imperial College London

$4^{\text {th }}$ floor, Imperial Centre for Translational and Experimental Medicine, Hammersmith Hospital campus, Du Cane Road, London, W12 0NN, UK.

kt112@imperial.ac.uk 